\title{
Local delivery of temozolomide via a biologically inert carrier (Temodex) prolongs survival in glioma patients, irrespectively of the methylation status of MGMT
}

\author{
I. KARLSSON ${ }^{1, *}$, D. VEEVNIK ${ }^{2}$, A. FEDULOV ${ }^{3}$, N. YURKSHTOVICH ${ }^{4}$, T. YURKSHTOVICH ${ }^{4}$, G. PEJLER ${ }^{5,6}$, I. LOKOT $^{1}$ \\ ${ }^{1}$ Double Bond Pharmaceutical AB, Uppsala, Sweden; ${ }^{2}$ Health Care Facility, Minsk City Emergency Clinical Hospital, Minsk, Belarus; ${ }^{3}$ Depart- \\ ment of Neurology and Neurosurgery, Belarusian State Medical University, Minsk, Belarus; ${ }^{4}$ Research Institute for Physical Chemical Problems, \\ Belarusian State University, Minsk, Belarus; ${ }^{5}$ Department of Medical Biochemistry and Microbiology, Uppsala University, Uppsala, Sweden; \\ ${ }^{6}$ Department of Anatomy, Physiology and Biochemistry, Swedish University of Agricultural Sciences, Uppsala, Sweden
}

*Correspondence: iulia@doublebp.com

Received June 13, 2018 / Accepted August 28, 2018

\begin{abstract}
Glioma is the most common brain malignancy. Standard first-line therapy for glioma includes surgery, radiotherapy and systemic administration of temozolomide. However, temozolomide does not reach the brain in sufficient doses when administered orally and has poor efficiency in more than half of the patients. Strategies to improve the treatment of glial malignancies are therefore needed. We have recently developed a system (Temodex) for local administration of temozolomide by encapsulating the drug in a biologically inert matrix. Here, we assessed the effect of Temodex in combination with standard therapy in a small-scale clinical study. Since the efficacy of temozolomide therapy is known to depend on the methylation status of the $\mathrm{O}^{6}$-methylguanine-DNA methyltransferase gene (MGMT) promoter, we also analyzed whether the effect of Temodex was influenced by the methylation status of MGMT. Our data show that the combination of standard therapy and Temodex was more efficient than standard therapy alone, promoting the overall patient survival by up to 33 weeks. Moreover, the efficacy of Temodex was not dependent on the methylation status of MGMT. Local Temodex administration in combination with standard therapy thereby emerges as a novel therapeutic option, with applicability that is independent on the methylation status of the MGMT promoter.
\end{abstract}

Key words: glioma, Temodex, temozolomide, MGTM, methylation

Gliomas constitute the most common form of brain tumours in adults. Despite the considerable efforts that have been invested into the development of improved therapeutic regimens for glioma, these malignancies are continuing to be associated with a notoriously poor prognosis [1]. Therefore, regimens to prolong survival in this disease are urgently needed. The standard therapy for glioma (Stupp therapy) includes surgery, radiotherapy and systemic administration of temozolomide [2]. Although the penetration of temozolomide into brain from plasma is better compared to other drugs, it is still limited, with the ratio of maximum plasma over brain tumor concentration of temozolomide after an oral administration being below 20\% [3]. Moreover, to achieve therapeutic levels in brain, temozolomide must be administered in high systemic doses due to its short halflife $(\sim 1.8 \mathrm{~h}$ in plasma [4]), which in turn requires prolonged systemic administration and leads to side effects such as thrombocytopenia, nausea and vomiting [5].
In order to achieve improved disease outcome, we have recently developed a system for sustained local delivery of temozolomide, by encapsulating the drug in a biologically degradable matrix. This system of temozolomide delivery (Temodex) has recently been approved for clinical use in Belarus (reg. number 14/12/2324, http://rceth.by/Refbank/ reestr_lekarstvennih_sredstv/results).

Previous studies have suggested that the outcome of temozolomide therapy is dependent on the methylation status of the $\mathrm{O}^{6}$-methylguanine-DNA methyltransferase gene (MGMT) $[6,7]$. MGMT is an enzyme that is crucial for repairing DNA damage, including damage caused by the genotoxic effects of chemotherapeutic drugs such as temozolomide. Hence, patients with active MGMT transcription in their tumor tissue are known to be relatively resistant to temozolomide effects whereas patients where MGMT transcription has been silenced by hypermethylation of the MGMT promoter are sensitive [8-10]. 
In this study, the aim was to evaluate the impact of Temodex on the clinical outcome of glioma and to determine if the effect of Temodex is dependent on the MGMT promoter methylation status. For this purpose, we studied the Temodex effect in a limited cohort of patients whose MGMT methylation status and MGMT protein expression had been characterized. Our results indicate a positive effect of Temodex and that its positive effect on overall survival may be independent of the MGMT promoter methylation status.

\section{Patients and methods}

Study setup. Paraffin-embedded tumor tissue samples were collected from patients with brain tumors during Temodex clinical trials at Emergency Hospital No.\#5 in Minsk, Belarus. The study was performed according to the ethical approval in protocol No.\#14 from 2012.07.30 issued by the hospital ethical committee, and the study protocol including patient informed consent and data and samples treatment was performed according to the Declaration of Helsinki. The samples were assessed for MGMT promoter methylation status and MGMT protein content. The analyses were blinded and were performed at Rigshospitalet (Copenhagen, Denmark). A total of 100 samples from 78 patients were analyzed, 29 patients received both standard care Stupp therapy of surgery, radiotherapy and systemic temozolomide (100 mg/patient) and Temodex (Temodex group; $900 \mathrm{mg} /$ patient), while the 49 control patients received only standard of care Stupp therapy (Control group). Temodex is temozolomide encapsulated in a biodegradable matrix. Upon contact with an aqueous milieu, Temodex forms a viscous gel that retains temozolomide at the administration site, thus allowing slow release and ensuring that wound edges are continuously exposed to temozolomide. Samples from the Temodex patient group were collected between November 2012 and December 2013, and samples from the control group were taken between October 2009 and October 2012. Patients were followed for up for 36 to 60 months and the overall survival of the cohort was assessed. Temodex has been approved for clinical use in Belarus since October 2014.

Sample preparation. Paraffin blocks were first re-paraffinized into new blocks and then sectioned for analyses: $4 \mu \mathrm{m}$ for Hematoxylin and Eosin (H\&E) staining and MGMT protein staining. The adjacent $30-50 \mu \mathrm{m}$ of the tissue was used for DNA extraction and MGMT gene promoter methylation analyses.

MGMT promoter methylation assessment. Sodium bisulfite conversion of the samples was performed using the Epitect bisulfite kit (Qiagen, Hilden, Germany) and PCR and pyrosequencing were performed using the Therascreen MGMT Pyro kit (Qiagen) according to the manufacturer's instructions. Three controls were included for each pyrosequencing run: a non-template control (NTC) from PCR, a methylated DNA control provided with the kit and one non-methylated DNA sample from an anonymous healthy blood donor. For assigning patients into a group of hypermethylated or non-methylated MGMT promoter status, two different thresholds were used: a mean methylation level of $8 \%$ [7] or $10 \%$ [11], i.e. methylation levels above either 8 or $10 \%$ were considered as hyper-methylation.

Immunohistochemistry. For immunohistochemistry analyses, formalin-fixed paraffin embedded sections were deparaffinized in xylene and rehydrated in decreasing ethanol concentrations. Endogenous peroxidase blocking with 3\% $\mathrm{H}_{2} \mathrm{O}_{2}$ was followed by pre-treating sections in a microwave oven with a Tris-EGTA-buffer and immune-staining on a DAKO Cytomation autostainer using monoclonal mouse anti-MGMT antibody (MAB16200, 1:200, Millipore, USA). Immunoreactivity was visualized with $\mathrm{DAB}+$ (DAKO K3468) as chromogen. The immunohistochemical stainings were semi-quantitatively evaluated according to the number of tumor cells stained: $0-25 \%=$ negative (methylated MGMT), and $>26 \%=$ positive (non-methylated MGMT). Positive endothelial cells, lymphocytes and microglia served as positive internal controls in the MGMT evaluation.

Statistical analysis. Baseline data was divided by MGMT status (gene methylation and immunohistochemistry) and assessed using Kaplan-Meier survival analyses (log-rank test) of the Temodex group compared to the control group, and of the patients that were MGMT hypermethylation-positive according to both DNA methylation grade and the IHC staining of the MGMT protein in the tumor tissue sections. For these analyses, Graph Pad Prism 4.0c software was used. Kaplan-Meier plots were used to present the outcome of the analyses. Any differences were considered statistically significant when the p-value was $<0.05$.

\section{Results}

The patient clinical data evaluated in this study are shown in Table 1. Notably, from the 78 patients, samples from 13 in the Temodex group and 26 in the control group were assessable for both MGMT methylation and tumor tissue protein expression (Supplementary Table 1). Representative staining for MGMT protein is shown in Figure 1. Data was further evaluated for patients in which MGMT protein was assessed and where also the MGMT promoter methylation status could be determined. In the Temodex group, DNA from 8 samples was positive for $M G M T$ promoter methylation, whereas DNA from 5 samples was non-methylated. In the control group, DNA from 19 samples was positive for MGMT promoter methylation whereas 7 were non-methylated.

Patients were treated with either standard therapy (surgery, radiotherapy, systemic temozolomide) or with a combination of standard therapy and a single dose of locally administered temozolomide (Temodex). The patients involved in these analyses had all been classified according to MGMT methylation status, as judged by both DNA methylation levels (see also below) and MGMT protein positivity. MGMT protein positivity is a sign of non-methylated MGMT promoter 
Table 1. Clinical and demographic characteristics.

\begin{tabular}{lcc}
\hline & $\begin{array}{c}\text { Study group } \\
(\mathbf{n}=\mathbf{2 9})\end{array}$ & $\begin{array}{c}\text { Control group } \\
(\mathbf{n}=\mathbf{4 9})\end{array}$ \\
\hline Males (\%) & $44.8(13)$ & $28.6(14)$ \\
Females (\%) & $55.2(16)$ & $71.4(35)$ \\
Age (years) & $57 \pm 12.1$ & $53 \pm 13.2$ \\
Pathomorphology and tumor & & \\
malignancy grade (\%) & & \\
$\quad$ Fibrillary astrocytoma, Grade II & $10.3(3)$ & $8.2(4)$ \\
Oligodendroglioma, Grade II & $6.9(2)$ & $6.1(3)$ \\
Oligoastrocytoma, Grade II & $0.0(0)$ & $4.1(2)$ \\
Anaplastic astrocytoma, Grade III & $3.5(1)$ & $4.1(2)$ \\
$\quad$ Glioblastoma, Grade IV & $79.3(23)$ & $77.5(38)$ \\
Prevalence in relation to & & \\
compartments (\%) & & \\
Subcortical (convexity) & $65.5(19)$ & $36.7(18)$ \\
Paraventricular & $17.2(5)$ & $30.6(15)$ \\
Mediobasal & $17.2(5)$ & $32.7(16)$ \\
\hline Karnofsky Performance & & \\
Status (\%) & & \\
100\% & $3.5(1)$ & $0.0(0)$ \\
$90 \%$ & $13.8(4)$ & $20.4(10)$ \\
$80 \%$ & $34.5(10)$ & $30.6(15)$ \\
$70 \%$ & $34.5(10)$ & $20.4(10)$ \\
$60 \%$ & $3.5(1)$ & $16.3(8)$ \\
$50 \%$ & $10.3(3)$ & $8.2(4)$ \\
$40 \%$ & $0.0(0)$ & $4.1(2)$ \\
\hline
\end{tabular}

whereas MGMT negativity is regarded as a sign of hypermethylated MGMT. The reason for evaluating both of these parameters is that previous studies have indicated that both of these parameters are important for determining treatment outcome $[8,12,13]$. MGMT protein positivity was assessed by immunohistochemical analyses (Figure 1).

Hypermethylation status and survival. The DNA hypermethylation threshold of the MGMT promoter was set at either $10 \%$ or $8 \%$, based on previous studies suggesting that either $10 \%$ [8] or $8 \%$ [14-16] can be considered relevant cut-offs in discriminating non- and hyper-methylated $M G M T$. Only samples where the MGMT methylation status was supported by both DNA analysis and by protein staining were included in the analyses.

We first analyzed the effect of Temodex by including all patients, i.e. both those displaying $M G M T$ hyper-methylation and those negative for MGMT hyper-methylation (Figure 2). When analysis was applied to patients with a $10 \%$ cut-off for DNA methylation, the results revealed that patients treated with Temodex in addition to standard of care had 14.2 weeks longer overall survival than those who received standard of care treatment alone $(\mathrm{p}=0.036$; Figure $2 \mathrm{~A})$. Under these conditions, the median overall survival was 41.36 weeks for the control group vs. 55.57 weeks for the Temodex group. Also when $8 \%$ was applied as the hyper-methylation cut-off, a pronounced effect of Temodex on overall survival was seen, with the Temodex treatment increasing the mean overall survival by 33 weeks ( $\mathrm{p}=0.027$, Figure $2 \mathrm{~B}$ ). In this setting, the median overall survival was 43.0 weeks for the
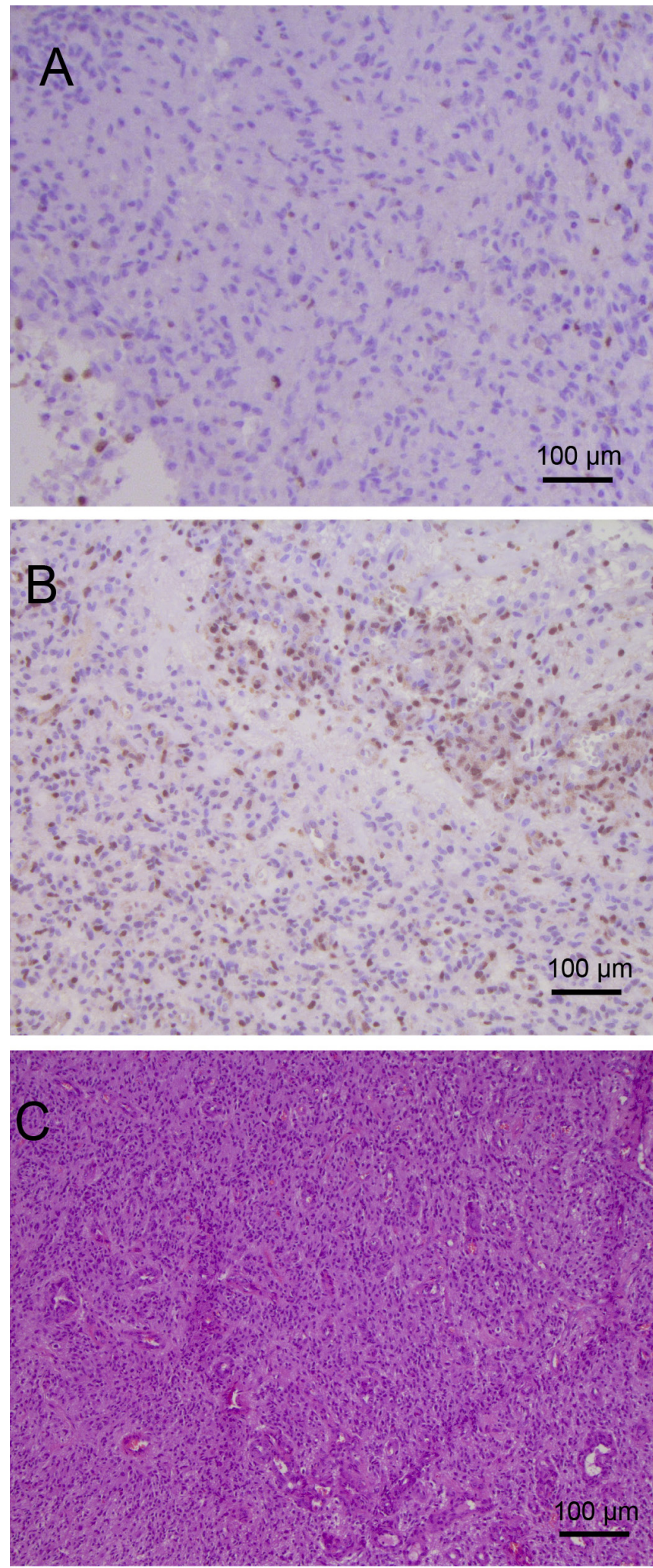

Figure 1. Immunhistochemical analysis of MGMT protein. Tissue sections from brain tumor samples were stained with MGMT antibody or with Hematoxylin and Eosin (H\&E). Representative image of MGMTnegative (A), MGMT-positive samples (B), and H\&E staining of representative MGTM-positive samples $(\mathrm{C})$ are shown. 

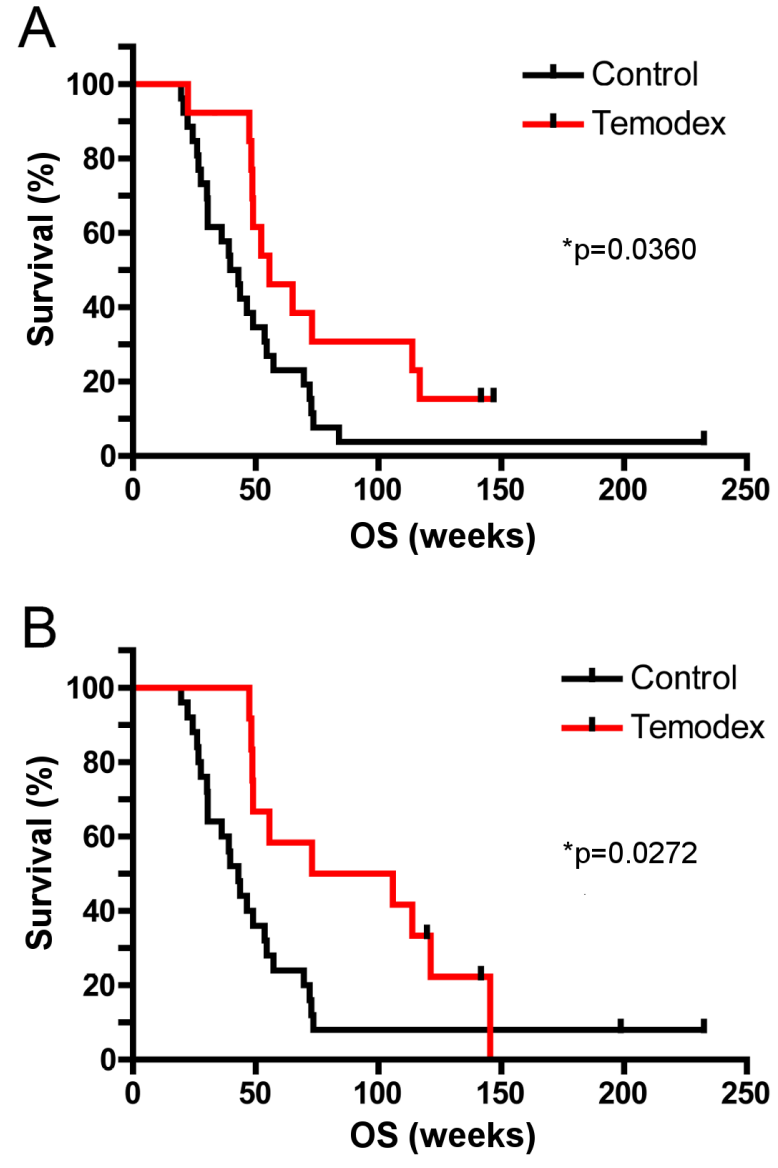

Figure 2. Kaplan-Meier survival plot for overall survival for patients treated with Temodex and standard of care (Temodex) compared to patients treated with standard of care only (Control). In these analyses, only patients where both the MGMT protein positivity and MGMT methylation status could be assessable, and where these parameters showed consistency, were included. The MGMT methylation threshold was set at over $10 \%$ (A) or $8 \%(B)$.

control group vs. 89.43 weeks for the Temodex group. Data from patients where only MGMT methylation status or only MGMT protein positivity could be assessed (not both) were also analyzed. In contrast to the patients where both of these parameters could be assessed, the Temodex treatment did not affect the outcome (data not shown).

Efficacy of Temodex treatment. To evaluate if the efficacy of Temodex treatment was dependent on the methylation status of MGMT, the effect of Temodex was evaluated on patients who had been discriminated into being positive or negative for MGMT hyper-methylation. Patients displaying MGMT hyper-methylation as judged by DNA analysis (with either $8 \%$ or $10 \%$ cut-off threshold) were denoted $\mathrm{MGMT}^{\mathrm{hm}+}$, and those where MGMT hyper-methylation was inferred by protein staining were denoted $\mathrm{MGMT}^{\mathrm{p}-}$. Patients negative for MGMT hyper-methylation were indicated as MGMT ${ }^{\mathrm{hm}-}$ and $\mathrm{MGMT}^{\mathrm{p}-}$, respectively.
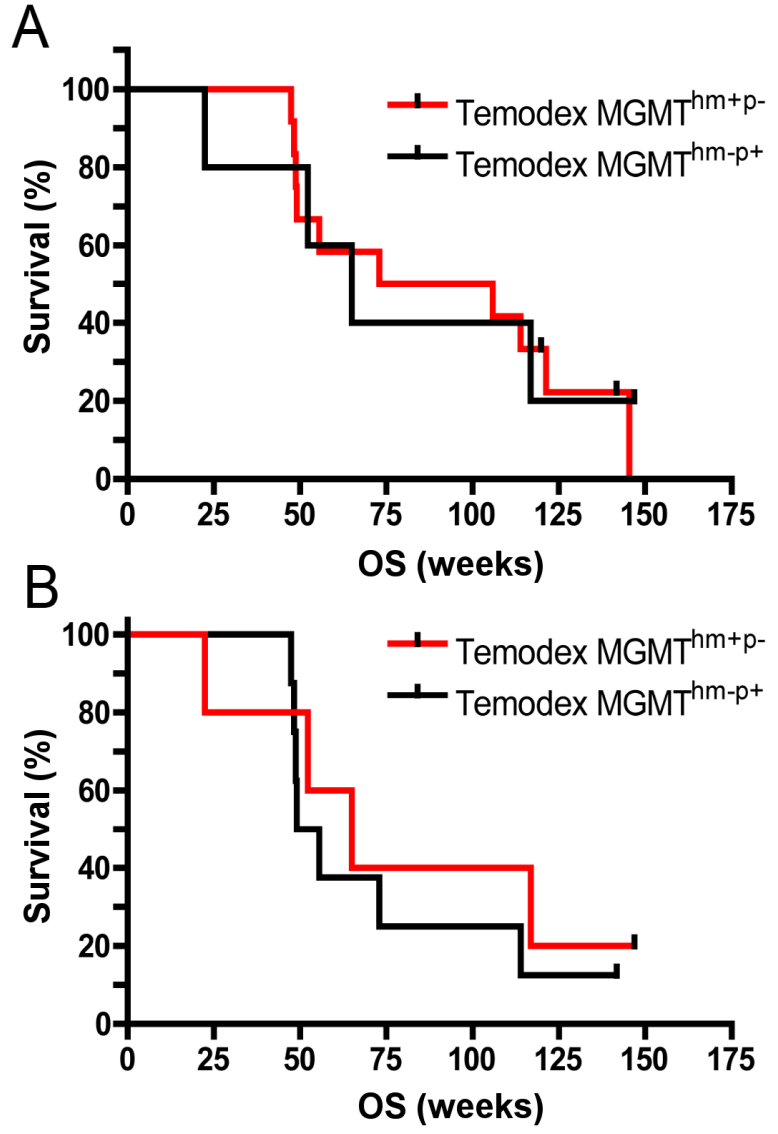

Figure 3. Kaplan-Meier survival plot for overall survival for patients treated with Temodex. Survival of patients positive for MGMT hypermethylation and negative for MGMT protein staining in the tumor tissue (Temodex MGMT $^{\mathrm{hm}+}$ ) did not differ significantly from the survival of patients without MGMT hyper-methylation and with positive MGMT protein staining in the tumor tissue (Temodex MGMT $^{\mathrm{hm}-}$ ). The thresholds for hyper-methylation were set at either $10 \%$ (A) or $8 \%$ (B).

Among patients treated with Temodex, there were no significant differences in overall survival between patients positive for MGMT promoter hyper-methylation according to both DNA analysis ( $\geq 10 \%$ methylation cut-off) and protein staining results $\left(\mathrm{MGMT}^{\mathrm{hm}+\mathrm{p}-}\right)$ compared to patients negative for MGMT methylation (MGMT ${ }^{\mathrm{hm}-\mathrm{p}+}$ ) (Figure 3A). When the threshold for hyper-methylation of the MGMT promoter was set to $\geq 8 \%$, similar results were obtained, i.e. Temodex had a positive effect on overall survival of patients irrespectively of the MGMT promoter methylation status (Figure 3B). Hence, Temodex has a positive effect on overall survival of patients regardless of the methylation status of MGMT and irrespectively if the cut-off for hyper-methylation is set at $8 \%$ or $10 \%$.

Data from patients where only MGMT methylation status or MGTM protein positivity had been considered were assessed for differences in survival between control groups 
(hyper-methylated vs. non-methylated), Temodex groups (hyper-methylated vs. non-methylated), non-methylated groups (Temodex vs. control) and hyper-methylated groups (control vs. Temodex). Neither of these analyses revealed statistically significant differences between the groups, either at $8 \%$ or $10 \%$ cut-off for MGMT methylation status (data not shown).

\section{Discussion}

Glioma continues to constitute a major pathology of the brain, afflicting a large number of subjects worldwide. Moreover, there are currently few effective therapeutic options available for this type of malignancy. In this study of relatively limited power, we show that the application of Temodex in combination with standard therapy has a profound and positive effect on the overall survival of glioma patients, with an increased survival time of up to 33 weeks. Thereby, the establishment of Temodex has expanded the repertoire of available therapeutic options in glioma, and we envision that Temodex may gain widespread use in the treatment of this malignancy. Moreover, we foresee that Temodex also may become useful in the treatment of other types of brain malignancies.

Intriguingly, we demonstrate that Temodex shows efficacy that is not dependent on the methylation status of the MGMT promoter, i.e. that Temodex is equally effective in patients with low or high expression of MGMT. This was somewhat unexpected considering previous studies indicating that temozolomide has low efficacy in patients with active expression of $M G M T$ as result of a low extent or absence of MGMT promoter hyper-methylation [17-19]. The underlying reason for the latter notion is that high $M G M T$ expression results in efficient DNA repair such that the genotoxic effects of temozolomide are minimized [6]. Although we cannot at present with certainty explain why Temodex shows high efficacy even in those patients with high MGMT expression, it should be emphasized that Temodex is directly applied at the tumor lesion site, and that the local concentration of temozolomide thereby will be considerably higher than when only administrating temozolomide systemically. We may thus propose that such high, local concentrations of temozolomide may impose more potent and rapid cytotoxic effects on the tumor cells than if the drug is merely administered systematically. This may lead to rapid induction of apoptosis such that the tumor cells fail to produce MGMT at levels that are sufficient to carry out efficient DNA repair.

It should be noted that the present study was performed on a relatively small patient cohort, and that extended studies on larger patient materials need to be carried out to firmly establish Temodex as a robust treatment option in glioma. Nevertheless, the present study establishes Temodex as a potential novel therapeutic agent to be used in combination with standard therapy in treatment of glioma patients showing both high and low MGMT expression. It is also notable that the Temodex represents a relatively cheap treatment option, and we therefore foresee that Temodex may become a preferred choice regardless of economic considerations.

Supplementary information is available in the online version of the paper.

Acknowledgements: We are grateful to Emergency Hospital No.\#5 (Minsk, Belarus) for providing samples for this study.

\section{References}

[1] CROCETTI E, TRAMA A, STILLER C, CALDARELLA A, SOFFIETTI R et al. Epidemiology of glial and non-glial brain tumours in Europe. Eur J Cancer 2012; 48: 1532-1542. http://doi.org/10.1016/j.ejca.2011.12.013

[2] STUPP R, BRADA M, VAN DEN BENT MJ, TONN JC, PENTHEROUDAKIS $G$ et al. High-grade glioma: ESMO Clinical Practice Guidelines for diagnosis, treatment and follow-up. Ann Oncol 2014; 25 Suppl 3: iii93-101. http://doi. org/10.1093/annonc/mdu050

[3] PORTNOW J, BADIE B, CHEN M, LIU A, BLANCHARD $S$ et al. The neuropharmacokinetics of temozolomide in patients with resectable brain tumors: potential implications for the current approach to chemoradiation. Clin Cancer Res 2009; 15: 7092-7098. http://doi.org/10.1158/1078-0432. CCR-09-1349

[4] BAKER SD, WIRTH M, STATKEVICH P, REIDENBERG P, ALTON K et al. Absorption, metabolism, and excretion of 14C-temozolomide following oral administration to patients with advanced cancer. Clin Cancer Res 1999; 5: 309-317.

[5] LAQUINTANA V, TRAPANI A, DENORA N, WANG F, GALLO JM et al. New strategies to deliver anticancer drugs to brain tumors. Expert Opin Drug Deliv 2009; 6: 10171032. http://doi.org/10.1517/17425240903167942

[6] HEGI ME, DISERENS AC, GORLIA T, HAMOU MF, DE TRIBOLET $\mathrm{N}$ et al. MGMT gene silencing and benefit from temozolomide in glioblastoma. N Engl J Med 2005; 352: 997-1003. http://doi.org/10.1056/NEJMoa043331

[7] MCDONALD KL, RAPKINS RW, OLIVIER J, ZHAO $\mathrm{L}$, NOZUE $\mathrm{K}$ et al. The $\mathrm{T}$ genotype of the MGMT $\mathrm{C}>\mathrm{T}$ (rs16906252) enhancer single-nucleotide polymorphism (SNP) is associated with promoter methylation and longer survival in glioblastoma patients. Eur J Cancer 2013; 49: 360-368. http://doi.org/10.1016/j.ejca.2012.08.012

[8] KRISTENSEN LS, MICHAELSEN SR, DYRBYE H, ASLAN D, GRUNNET K et al. Assessment of Quantitative and Allelic MGMT Methylation Patterns as a Prognostic Marker in Glioblastoma. J Neuropathol Exp Neurol 2016; 75: 246-255. http://doi.org/10.1093/jnen/nlv024

[9] TANG K, JIN Q, YAN W, ZHANG W, YOU G et al. Clinical correlation of MGMT protein expression and promoter methylation in Chinese glioblastoma patients. Med Oncol 2012; 29: 1292-1296. http://doi.org/10.1007/s12032-0119901-4 
[10] WELLER M, TABATABAI G, KASTNER B, FELSBERG J, STEINBACH JP et al. MGMT Promoter Methylation Is a Strong Prognostic Biomarker for Benefit from Dose-Intensified Temozolomide Rechallenge in Progressive Glioblastoma: The DIRECTOR Trial. Clin Cancer Res 2015; 21: 2057-2064. http://doi.org/10.1158/1078-0432.CCR-14-2737

[11] XIE H, TUBBS R, YANG B. Detection of MGMT promoter methylation in glioblastoma using pyrosequencing. Int J Clin Exp Pathol 2015; 8: 636-642.

[12] THON N, KRETH S, KRETH FW. Personalized treatment strategies in glioblastoma: MGMT promoter methylation status. Onco Targets Ther 2013; 6: 1363-1372. http://doi. org/10.2147/OTT.S50208

[13] WELLER M. Assessing the MGMT status in glioblastoma: one step forward, two steps back? Neuro Oncol 2013; 15: 253-254. http://doi.org/10.1093/neuonc/not014

[14] FELSBERG J, THON N, EIGENBROD S, HENTSCHEL $\mathrm{B}, \mathrm{SABEL} \mathrm{MC}$ et al. Promoter methylation and expression of MGMT and the DNA mismatch repair genes MLH1, MSH2, MSH6 and PMS2 in paired primary and recurrent glioblastomas. Int J Cancer 2011; 129: 659-670. http://doi. org/10.1002/ijc.26083

[15] REIFENBERGER G, HENTSCHEL B, FELSBERG J, SCHACKERT G, SIMON M et al. Predictive impact of MGMT promoter methylation in glioblastoma of the elderly. Int J Cancer 2012; 131: 1342-1350. http://doi.org/10.1002/ijc.27385
[16] SWITZENY OJ, CHRISTMANN M, RENOVANZ M, GIESE A, SOMMER C et al. MGMT promoter methylation determined by HRM in comparison to MSP and pyrosequencing for predicting high-grade glioma response. Clin Epigenetics 2016; 8: 49. http://doi.org/10.1186/s13148-016-0204-7

[17] BRELL M, TORTOSA A, VERGER E, GIL JM, VINOLAS $\mathrm{N}$ et al. Prognostic significance of O6-methylguanine-DNA methyltransferase determined by promoter hypermethylation and immunohistochemical expression in anaplastic gliomas. Clin Cancer Res 2005; 11: 5167-5174. http://doi. org/10.1158/1078-0432.CCR-05-0230

[18] HEGI ME, DISERENS AC, GODARD S, DIETRICH PY, REGLI $L$ et al. Clinical trial substantiates the predictive value of O-6-methylguanine-DNA methyltransferase promoter methylation in glioblastoma patients treated with temozolomide. Clin Cancer Res 2004; 10: 1871-1874. http://doi. org/10.1158/1078-0432.CCR-03-0384

[19] ZHANG K, WANG XQ, ZHOU B AND ZHANG L. The prognostic value of MGMT promoter methylation in Glioblastoma multiforme: a meta-analysis. Fam Cancer 2013; 12: 449-458. http://doi.org/10.1007/s10689-013-9607-1 